\title{
Early results of a Phased Array Feed system at Effelsberg
}

\author{
Xinping Deng ${ }^{1,2}$, Aaron Chippendale ${ }^{2}$, Ewan Barr ${ }^{1}$, \\ Mateusz Malenta ${ }^{3}$, Olaf Wucknitz ${ }^{1}$, Guðjón Henning Hilmarsson ${ }^{1}$, \\ Leon Houben ${ }^{1}$, Daniel George ${ }^{2}$, Laura Spitler ${ }^{1}$, Ramesh \\ Karuppusamy $^{1}$, Michael Kramer ${ }^{1,3}$ and Gundolf Wieching ${ }^{1}$ \\ ${ }^{1}$ Max-Planck-Institut für Radioastronomie, \\ Auf dem Hügel 69, 53121 Bonn, Germany, \\ ${ }^{2}$ CSIRO Astronomy and Space Science, Australia Telescope National Facility, \\ P.O. Box 76, Epping NSW 1710, Australia, \\ 3 Jodrell Bank Centre for Astrophysics, The University of Manchester, \\ Alan Turing Building, Manchester M13 9PL, UK
}

\begin{abstract}
The overview of a Phased Array Feed (PAF) system and the early results with it on the $100 \mathrm{~m}$ diameter telescope at Effelsberg are presented in the paper.
\end{abstract}

Keywords. instrumentation: detectors, (stars:) pulsars: individual (PSR J0358+5413), etc.

\section{Introduction}

In a collaboration, the Australia Telescope National Facility and the Max-PlanckInstitut für Radioastronomie have embarked on a project to install and utilize a Phased Array Feed (PAF) system on large-gain, single-dish telescope with significant direct access for astronomers.

After the initial commissioning observations on the Parkes $64 \mathrm{~m}$ telescope, the system was shipped to Effelsberg and tested in Spring this year. In order to commission it, we re-installed it in the primary focus of Effelsberg $100 \mathrm{~m}$ telescope from $3^{\text {rd }}$ to $10^{\text {th }}$ August. Here we give an overview of the system and represent two early observations $\dagger$ we did with it at that time.

\section{System overview}

A PAF receiver is a dense array of antenna elements at the focus of a reflector telescope and the output of these elements can be combined to form beams on the sky. The direction of these beams is controlled by varying the weights of individual elements. The PAF (Hampson et al. 2012) described here was designed for the Australian Square Kilometre Array Pathfinder (ASKAP) telescope, but slightly modified for use on the Effelsberg telescope (Chippendale et al. 2016).

An array of 188 connected "chequerboard" antenna elements is distributed over approximately a $1.2 \mathrm{~m}$ diameter circle. It is a dual-polarisation receiver and each polarisation has 94 elements. The analog signals from all elements, each of up to $600 \mathrm{MHz}$ bandwidth, are transmitted to the digital receiver via RF-over-fibre links and sampled there by 12 "Dragonfly" digital receivers. The digital receivers also channelize the data to $1 \mathrm{MHz}$ via a multi-stage oversampled filterbank (Tuthill et al. 2012). With 16 ports per receiver this results in a 192-port digital system, with four spare ports beyond the 188 connected to

$\dagger$ Please look at the poster for the observations which we can not present in the paper. 

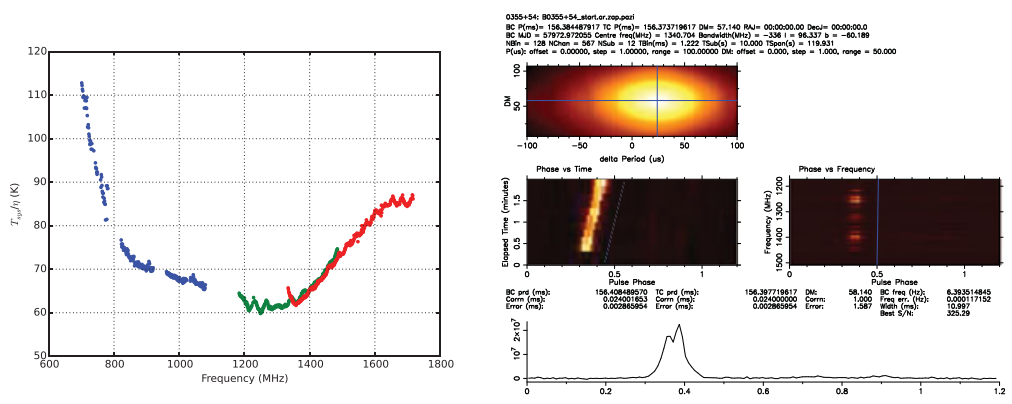

Figure 1. Early results of a PAF system at Effelsberg $100 \mathrm{~m}$ telescope. The left sub-figure represents the system temperature over efficiency of PAF's central beam. From left to right are the results for $1200 \mathrm{MHz}, 1450 \mathrm{MHz}$ and $1800 \mathrm{MHz}$ bands separately. The right sub-figure shows the observation of PSR J0358+5413 (B0355+54) with the same beam.

the PAF receiver. The digitised signals are processed by eight "Redback" beamformers to form up to 36 dual-polarisation beams of $384 \mathrm{MHz}$ bandwidth (48 $\mathrm{MHz}$ per beamformer) in $1 \mathrm{MHz}$ frequency channels. We stream $336 \mathrm{MHz}$ of 16 -bit beamformed baseband data (42 MHz per beamformer) at the full sampling rate into Graphics Processing Unit (GPU) nodes via Ethernet switches in $7 \mathrm{MHz}$ frequency chunks. The beamformed data is converted to filterbank format files on these GPU nodes for further processing.

\section{Observations}

We measured the system temperature over efficiency of PAF's central beam with on and off-source observations of Cas A. The left sub-figure of Figure 1 is the result with Radio Frequency Interference (RFI) channels manually removed. We can see that the system temperature over efficiency is around $70 \mathrm{~K}$ for the central band. It increases towards the edges of band, with much better performance between $800 \mathrm{MHz}$ to $1400 \mathrm{MHz}$.

We used the same beam to observe strong pulsars to check the behaviour of the endto-end system. The right sub-figure of Figure 1 shows one of these observations (the observation of PSR J0358+5413, which is also known as PSR B0355+54).

\section{Conclusion}

We installed a PAF system on a large-gain, single-dish telescope. We successfully measured the system temperature over efficiency of it and observed strong pulsars to check the behaviour of it.

\section{Acknowledgements}

This project represents the combined efforts of a large number of people. We are especially grateful to the highly motivated and skilled staff at Effelsberg.

\section{References}

Hampson, G., Macleod, A., Beresford, R., Brothers, M., Brown, A., Bunton, J., Cantrall, C., Chekkala, R., Cheng, W., \& Forsyth, R. and Gough, R 2012, ICEAA, 807-809

Chippendale AP, Beresford RJ, Deng X, Leach M, Reynolds JE, Kramer M, \& Tzioumis T. 2016 ICEAA, 909-912

Tuthill, J., Hampson, G., Bunton, J., Brown, A., Neuhold, S., Bateman, T., de Souza, L., \& Joseph, J. 2012 ICEAA, 1067-1070 